Sharon Dodua Otoo

\title{
The Story of Circle and Quadrangle
}

\author{
Translated by Özlem Karuç
}



Once upon a time there was a circle that lived in a community of quadrangles. The circle spoke like the quadrangles and behaved like them. It was startling: it could even pretend to be a quadrangle whenever the situation called for it - which in fact happened very often. Sometimes it was enough for the circle to simply say nothing. Whenever the values of the quadrangles, such as being angular, were passionately held up high, short but clear lip service was advantageous. The circle always delivered that, in a well-behaved way, too, without wanting to contemplate much. Gradually, of course, the quadrangles stopped paying attention to the otherness of the circle. Its roundness was barely mentioned. It did not have to be. The quadrangles regarded the circle as "one of us."

The circle was well informed about the history of the quadrangles, about their attributes too. It knew, for example, that the sum of all the angles in a quadrangle equals 360 degrees, that all four sides were the same length, that all quadrangles had four right angles and four axes of symmetry, and that their diagonals were the same length, perpendicular to one another, and always halved. In fact, it was more of an expert on quadrangles than the quadrangles themselves. For the circle knew that they were really called "squares" and that there were actually other quadrangles that had different attributes. Why the other quadrangles in the community were not recognizable was rarely discussed. And neither was why the squares had occupied the name "quadrangle" for themselves alone. And that was probably why life was so good there.

One day another circle appeared in the community of quadrangles - a circle that did not disguise itself. Maybe it did not know that it should have done that. Or, and this is more likely, while it was meticulously well informed about the conditions of the community, it refused to apply the strategies of the first circle. Or continue to use them. In any case, it threatened to take forever for the two circles to find the courage and trust to meet-but eventually they managed it after all.

At some point, they encountered each other, each recognized itself in the other-along with their beautiful scars, along with their perfect quirks. For the first time in their lives, the two circles could just be themselves: complete and vulnerable. 
They talked about a lot of things, and their shared vocabulary expanded to include wonderful new words such as "round" and "smooth." The word "corner" was not mentioned once. The circles rolled on the floor laughing until late into the night, until their edges trembled, until they could not cry anymore.

Consequently, the quadrangles wanted to get an easy-tounderstand explanation the next day for why the circles had made so much noise during the night. The first circle tried to explain the matter with "round" and "smooth," but the quadrangles just looked aghast. The second circle twirled several times to depict the whole thing. But the quadrangles were still incapable of understanding, for the comfort of not-wanting-to-know is seductively and easily confused with a transient, superficial confusion. And since the circles would never again be like they had always been before, there was the threat of a split in the community. Many a square tear was shed.

Fortunately, there was a plan B. One of the quadrangles had traveled abroad once and discovered a completely new community of forms that called itself "triangles." Since the claim was that the circles were also somehow "different" and recognizably came from "somewhere else," they should be called "weird triangles." Even the circles themselves were happy with the new name. Too bad that they could not come up with a name for themselves on such short notice, but at least it was finally recognized that they had their own shape-identity.

Years later, in a community where many different shapes lived (of which most were still quadrangles), the "weird triangles" began to realize that the name "weird triangles" was actually completely wrong. They were not "weird triangles" but circles. And they were not "angle-less" or "non-angular" but "round" and "smooth." The debate over the new name was tedious. Many quadrangles made fun of the "weird triangles" and dismissed the whole thing as wordplay. They said that it did not matter what the words were as long as it was agreed that everyone meant the same thing with the words. Other quadrangles clamped onto the fact that the name "weird triangle" had come into being at a time when the word "weird" was not offensive and therefore could not be offensive now either. Some of the "weird triangles" were already too old or too aligned with the views of 
the quadrangles to care about these new-fangled circles. Why think about difference? All shapes were equal after all: all shape-lives mattered! Only a few of the quadrangles really took the circles seriously.

At some point in the distant future, circles, squares, triangles, quadrangles, rhombuses, and parallelograms will all live in a community in which everyone knows what "straight," "round," "curved," and "angular" mean. Each shape will have found a name for itself and will be able to communicate with one another without offending other shapes with its language. 